\title{
Basilea II y el crédito PyME en Colombia
}

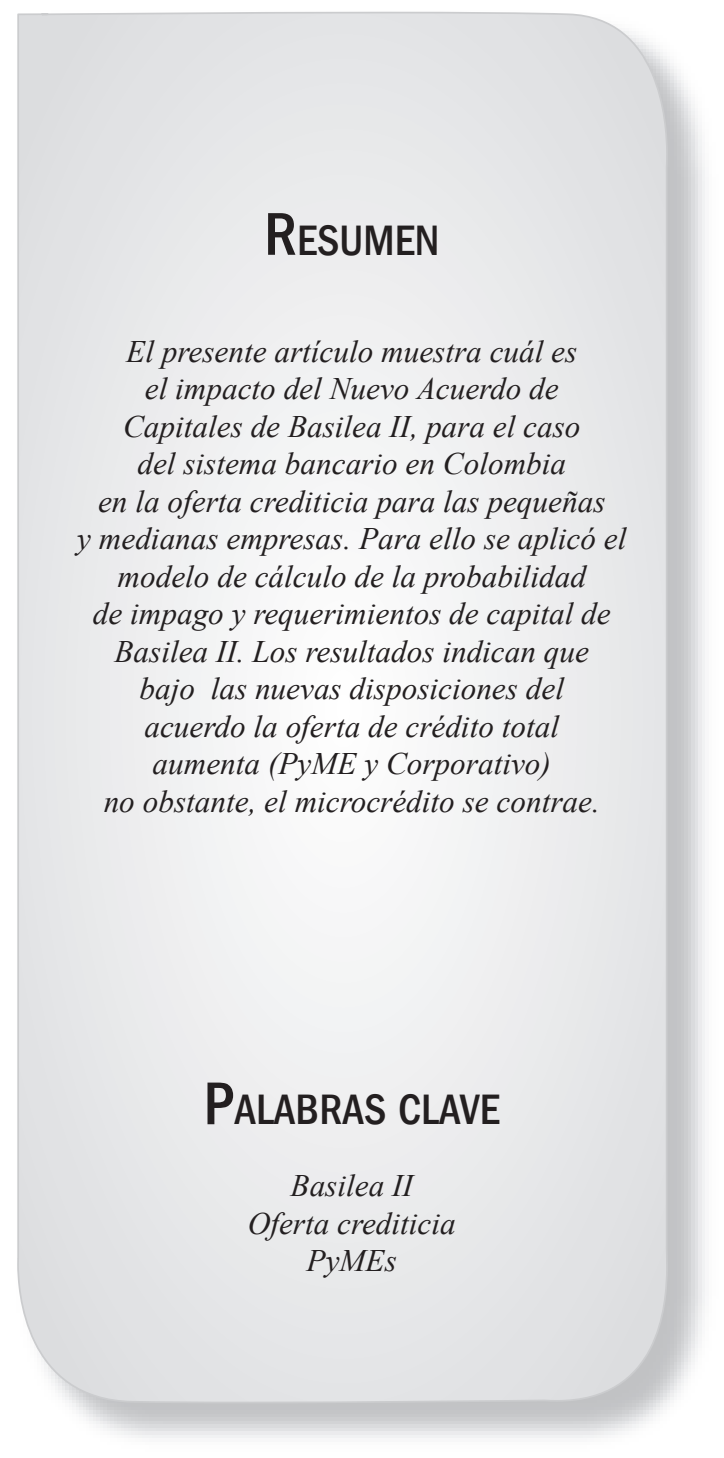

\section{Introducción}

El Acuerdo de Basilea surge como respuesta a la necesidad de proporcionar un marco de referencia institucional con el fin de lograr la estabilidad y seguridad del sistema financiero internacional, en un ámbito de economía global y de mercado. El primer acuerdo data de 1988 y fue creado por los bancos centrales de las principales economías del mundo, con la filosofía de ser aplicado libremente en cada uno de los países que decidieran implementarlo. En 2004, nace el Nuevo Acuerdo de Capitales (NAC) que busca subsanar las carencias del primero, principalmente en términos de calidad crediticia y valoración del riesgo de los bancos.

Colombia no se encuentra ajena a los alcances del NAC, la que trae consigo

* Economista de la Universidad Nacional de Colombia, Master en Finanzas de la Universidad de Alcalá de Henares España. Gerente de Proyectos de Capitalia Colombia. 
una serie de exigencias e implicaciones, haciendo necesario evaluar el impacto que supone la aplicación de éste en el sistema financiero. Al respecto, Saurina y Trucharte (2002) afirman que la implementación del acuerdo afectará de manera drástica a las economías de países emergentes, al reducir los flujos de capitales disponibles para financiar los diversos sectores de la economía y en particular a las pequeñas y medianas empresas (PyMEs), que históricamente han presentado las tasas más altas de morosidad en el sistema financiero.

En países como Estados Unidos y Japón las PyMEs constituyen la mayor fuente de generación de empleo. En el caso colombiano, su relevancia igualmente es significativa; el sector PyME representa el $91 \%$ del total de empresas formalmente establecidas; es responsable del $40 \%$ de la producción; del $30 \%$ del empleo y posee el $20 \%$ de las inversiones nacionales (DANE, 2005).

Por otra parte, si bien es cierto que entre 1999 y 2001 el segmento PyME presentó un declive en su ritmo de crecimiento, a partir de 2002 viene mostrando un comportamiento positivo acorde al crecimiento deI PIB nacional, lo cual indica una recuperación y aprovechamiento de las ventajas de acuerdos internacionales, especialmente con Estados Unidos; como por ejemplo la desgravación de productos de la oferta exportable y la adquisición de materia prima a costos más favorables. Su crecimiento también se ve reflejado

\section{Abstract}

This article shows the impact of the new agreement of capitals in BASILEA II in relation with the credit offer for PyMEs in the case of the banking Colombian system. The model of unpaid probability calculus and requirements of Basilea II capital were applied. The results show that under the lights of such agreement, there is an increase in the offer of total credits (PyME and corporative); however, the microcredit system tends to become smaller.

\section{KEy WORDS}

Basilea II

Credit offer

PyMEs

Global economy

Emerging economy 
en el aumento de créditos al sector; entre 2005 y 2006 , el crédito con garantías del Fondo Nacional de Garantías a las PyMEs aumentó un $52 \%$, este mismo sector obtuvo un $47 \%$ de los desembolsos de Bancoldex ${ }^{1}$, de los cuales un $34 \%$ se destinó a modernización y un $28 \%$ a capital de trabajo, mientras que las grandes empresas recibieron un $25 \%$ (Clavijo, 2006).

Sin embargo, a pesar del alentador panorama, la banca percibe este segmento como riesgoso dado el bajo respaldo patrimonial que poseen y su débil estructura financiera, lo que restringe de manera significativa sus fuentes de financiación. El papel de la banca como agente financiador en Colombia, junto con los requerimientos de los mercados internacionales en cuanto al cumplimiento de Basilea II, da forma al escenario que los mercados financieros impondrán en mediano y largo plazo para este segmento, en una economía emergente y globalizada como la colombiana.
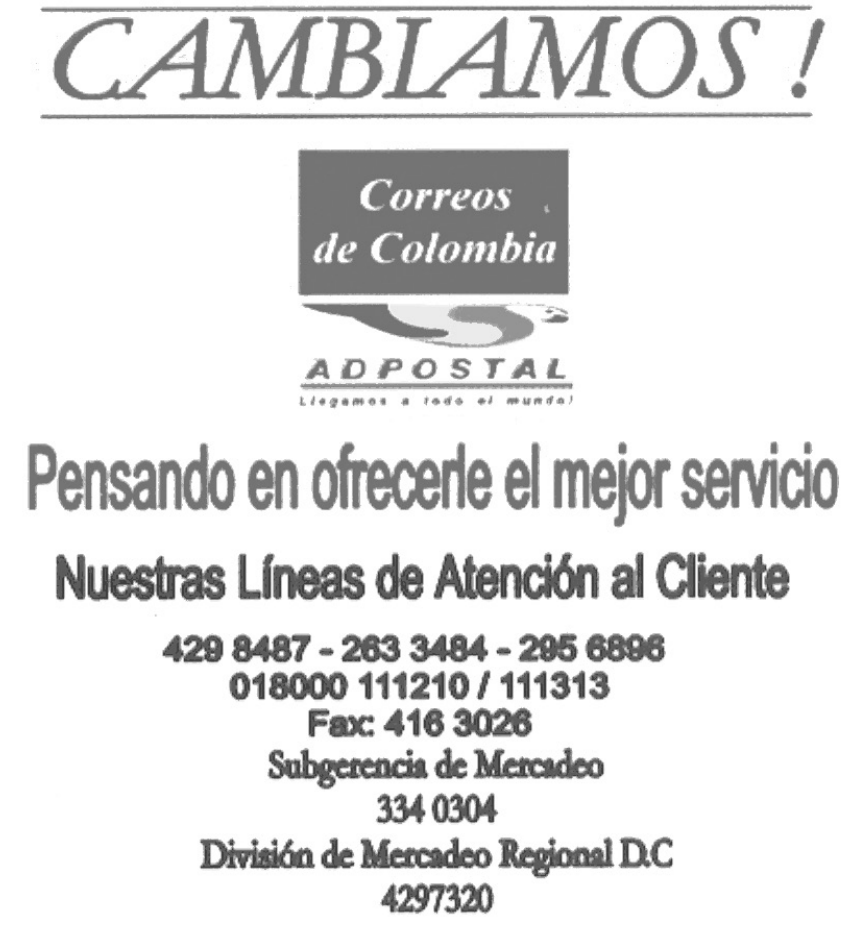

www.acpostal.gov.co

\footnotetext{
${ }^{1}$ El Fondo Nacional de Garantías y Bancoldex son entidades financieras públicas que funcionan como bancos de segundo piso, que cubren operaciones en las que las contrapartidas no posene el suficiente respaldo patrimonial para la operación.
} 


\section{Aproximación a la probabilidad de impago (pd) y requerimientos de capital en el crédito a las PyMEs en Colombia bajo Basilea II}

\subsection{Financiación otorgada al sector}

Del total de instituciones financieras, es el sector bancario el que concentra la mayor parte de créditos (Ver gráfica 1). Por ello, el presente análisis, se centra en el crédito bancario otorgado al segmento PyME.

Gráfica 1. Crédito por instituciones financieras 2008

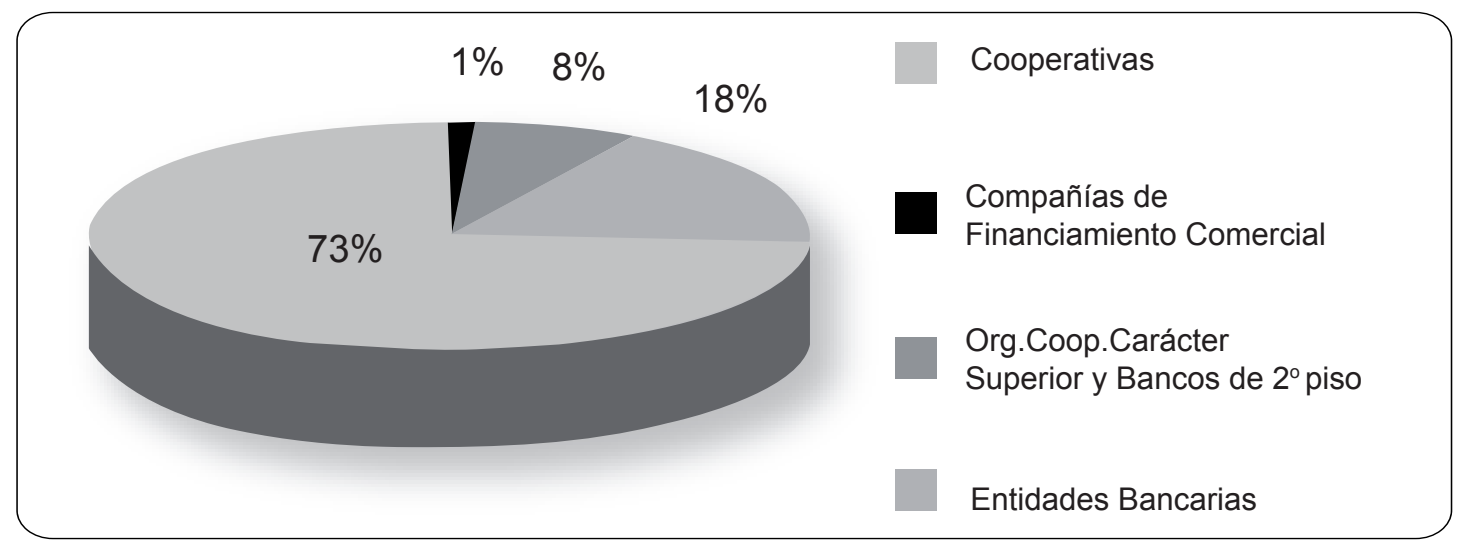

Fuente. Cálculos propios con datos de la Superintendencia Financiera de Colombia, 2008

El crédito al segmento PyME en las estadísticas colombianas se encuentra consolidado dentro del total comercial de los bancos. Es por ello, que para estimar la cartera bancaria PyME se han tomado las estadísticas de Sandoval (2007) de la participación PyME en la cartera comercial bancaria y se han multiplicado por la cartera comercial año a año de los bancos, disponible en la serie de indicadores gerenciales bancarios de la Superintendencia Financiera de Colombia (2008). Por su parte, la cartera MiPyME se encuentra discriminada como cartera microcrédito, un tipo dentro de las clasificaciones estándar: comercial, hipotecaria, consumo y microcrédito. 
En la Tabla 1, se presenta la evolución de la cartera de 2003 a 2007 por segmento empresarial y se observa que la cartera total ha presentado un crecimiento sostenido hasta 2007; año en que disminuye su tasa al ser comparada con la correspondiente al 2006. De forma similar, los segmentos PyME y MiPyME han evolucionado favorablemente durante los últimos años, evidenciando la importancia que tienen en el desarrollo en la bancarización nacional.

Tabla 1. Porcentaje de participación de la cartera bancaria PyME y MiPyME

\begin{tabular}{|c|c|c|c|c|c|c|}
\hline \multirow{2}{*}{ Año } & \multirow{2}{*}{\multicolumn{2}{|c|}{$\begin{array}{l}\text { Total cartera comercial + } \\
\text { MiPyME en MM de US }\end{array}$}} & \multicolumn{3}{|c|}{ \% Participación en cartera } & \multirow{2}{*}{$\begin{array}{c}\text { Tasa de } \\
\text { crecimiento } \\
\text { cartera total }\end{array}$} \\
\hline & & & & & & \\
\hline 2003 & $\$$ & 15,914 & $1.8 \%$ & $12.9 \%$ & $85.3 \%$ & -- \\
\hline 2004 & $\$$ & 18,438 & $2.4 \%$ & $19.9 \%$ & $77.7 \%$ & $15.9 \%$ \\
\hline 2005 & $\$$ & 21,938 & $3.0 \%$ & $21.2 \%$ & $75.8 \%$ & $19.0 \%$ \\
\hline 2006 & $\$$ & 29,497 & $3.0 \%$ & $25.7 \%$ & $71.3 \%$ & $34.5 \%$ \\
\hline \multirow[t]{2}{*}{2007} & $\$$ & 35,925 & $2.8 \%$ & $25.7 \%$ & $71.5 \%$ & $21.8 \%$ \\
\hline & Promedio & $2.6 \%$ & $21.1 \%$ & $76.3 \%$ & $22.8 \%$ & \\
\hline
\end{tabular}

Fuente. Cálculos propios con datos de la Superintendencia Financiera de Colombia, 2008 y Sandoval, 2007.

\subsection{Estimación del capital mínimo según Basilea II}

En este apartado se realiza una aproximación al sistema establecido por el Comité de Basilea con el fin de entender la probabilidad de fallidos y posteriormente el cálculo del capital mínimo.

La creación de modelos internos para la medición y control del riesgo, es hoy una prioridad para las entidades financieras. A partir de su uso, se obtiene la distribución de probabilidad de pérdidas potenciales en términos del riesgo crediticio de cada organización. Esta distribución resulta muy importante dado que permite, entre otras cosas, obtener la estructura de capital óptima para cubrir adecuadamente la exposición de una entidad ante posibles pérdidas por riesgo de crédito. En consecuencia, la correcta medición del riesgo de crédito dependerá fuertemente de la distribución de probabilidad de pérdidas esperadas.

El comité de Basilea, utiliza como base un modelo factorial de riesgo de crédito para calcular la distribución de probabilidad de pérdidas. La gran ventaja de utilizar este 
tipo de modelos reside en que permiten replicar comportamientos de impago a partir de la correlación que existe entre los acreditados. En resumen, este modelo describe la probabilidad de impago a partir de la probabilidad de que los activos de una empresa presenten valor inferior al de la deuda contraída.

\subsubsection{Función de probabilidad de fallidos}

Según Trucharte y Antuña (2001), la función de distribución acumulada para un determinado porcentaje de fallidos (x) está dada por:

$$
F(x)=\operatorname{Prob}\left[\frac{X}{M} \leq x\right]=N\left(\frac{1}{\sqrt{p}}\left(\sqrt{1-\rho N^{-1}(x)-N^{-1}(P D)}\right)\right)
$$

Donde,

$X$ : número de fallidos

$\rho$ : coeficiente de correlación de los acreditados.

PD: establece la probabilidad de impago a partir de la probabilidad de que el valor de las deudas $(\mathrm{K})$ de los acreditados superen el valor de los activos $(\mathrm{Vm})$ :

$$
\mathrm{PD}=\mathrm{PROB}(\mathrm{Vm}<\mathrm{K})
$$

A partir de esta ecuación se establece que:

$P D=N(K)$, y por tanto $K=N^{-1}(P D)$

Al derivar con respecto a $(x)$ se obtiene la función de densidad del porcentaje de fallidos de la cartera de crédito:

$$
F(x)=\sqrt{\frac{1-p}{p}} e^{\frac{1}{2}\left(N^{-1}(x)\right)^{2}-\frac{1}{2 p}\left(\sqrt{1-p} N^{-1}(x)-N^{-1}(P D)\right)^{2}}
$$

Con esta función se obtiene la expresión para la densidad de probabilidad de la función de pérdidas.

\subsubsection{Modelo establecido por Basilea para el cálculo de capital mínimo}

El documento consultivo de 2001 del Comité de Basilea se fundamenta en un modelo unifactorial. En este documento se plantea que el capital mínimo requerido debe calcularse para grupos homogéneos de riesgo, es decir, cada grupo debe tener características similares, especialmente en lo referente a la probabilidad de impago; para ello, divide la cartera en dos grandes grupos, cuya suma constituye la cartera crediticia global: 
D Grandes Exposiciones (Corporate Exposures).

D Pequeñas exposiciones (Retail Exposures).

Estos requerimientos establecen un capital mínimo del 8\% para cada exposición, ponderado por unos determinados pesos denominados Risk Weights (RW). Las ponderaciones son obtenidas a partir de unos Benchmark Risk Weights (BRW) los cuales se calculan a partir de una distribución de probabilidad de fallidos.

¿En qué consisten esos RW y BRW?

\section{Cartera Corporate Exposures}

Para el cálculo de los requerimientos de capital en la cartera Corporate se establecen ciertas convenciones en las variables de cálculo, las cuales se recogen en el siguiente apartado.

\section{- Convenciones para el cálculo del} capital requerido

El comité de Basilea establece que el nivel de probabilidad objetivo para determinar los requerimientos de capital (TSP) sea del 99.9\%.

En cuanto al factor de correlación entre los activos, este se establece a partir de la siguiente ecuación:

$$
R=0.12 * \frac{\left(1-\exp ^{\left(-50^{*} P D\right)}\right)}{\left(1-\exp ^{-50}\right)}+0.2 *\left[1-\frac{\left(1-\exp ^{(-50 * P D)}\right)}{1-\exp ^{-50}}\right]
$$

En lo referente a la maduración de la cartera, documentos consultivos previos como el de enero de 2001, establecía una maduración media de la cartera de tres años; sin embargo, esta característica fue modificada ya que ahora debe fijarse en función del tipo de cartera de cada entidad de crédito. Se incluye en el documento consultivo de octubre de 2002 un ajuste a la maduración a partir de la siguiente fórmula:

$$
\text { Maturity adjustment }(\mathrm{b})=(0.08451-0.05898 \times \log (P D))^{\wedge} 2
$$

\section{- Cálculo del capital mínimo requerido}

A partir de estas consideraciones (Comité de supervisión bancaria de Basilea, 2002), se establece la fórmula para el cálculo de capital requerido (K) así:

$$
K=L G D^{*} N\left[\left(1-R^{-0.5 *} G(P D)+\left(\frac{R}{(1-R)}\right)^{0.5} * G(0.999)\right] *\left(1-1.5^{*} b(P D)\right)^{-1} *\left(1+(M-2.5)^{*} b(P D)\right)\right.
$$


En esta ecuación, LGD representa el porcentaje de pérdida en caso de impago, el cual, según el documento consultivo de enero (2001), puede oscilar entre un 35\% y un $70 \%$, según lo estime cada institución. El cálculo de los Risk Weight se realiza a partir de la siguiente expresión:

$$
\mathrm{RW}=\mathrm{K} * 12.5 \text { * } \mathrm{EAD}
$$

\section{- Cartera Retail Exposures}

Para las exposiciones de menor tamaño se establecen algunas diferencias para el cálculo del capital mínimo en comparación con las Corporate Exposures.

\section{$>$ Convenciones para el cálculo del capital requerido}

El nivel de probabilidad objetivo para determinar los requerimientos de capital (TSP) es de igual manera del 99.9\%; sin embargo, el factor de correlación tiene algunas diferencias:

$$
R=0.02 * \frac{\left(1-\exp ^{(-50 * P D)}\right)}{\left(1-\exp ^{-50}\right)}+0.15 *\left[1-\frac{\left(1-\exp ^{(-50 * P D)}\right)}{1-\exp ^{-50}}\right]
$$

Además, no se incluyen ajustes por maduración de la cartera.

\section{$>$ Cálculo del capital mínimo requerido}

La expresión que establece el cálculo de capital requerido (Comité de Supervisión Bancaria de Basilea, 2002) es la siguiente:

$$
K=L G D * N\left[\left(1-R^{-0.5 *} G(D P)+\left(\frac{R}{(1-R)}\right)^{0.5} * G(0.999)\right]-0.9 P D * L G D\right.
$$

El cálculo de los Risk Weight se calcula de la misma manera que en las grandes exposiciones:

$$
R W=K * 12.5 * E A D
$$

Los requerimientos de capital para el total de la cartera se estiman a partir de la suma individual de los requerimientos de capital de cada grupo homogéneo en los que está dividida la cartera, establecidos como un $8 \%$ de cada una de los grupos ponderados por los RW que les corresponda. Los requerimientos mínimos de capital se calculan considerando características individuales de cada acreditado y no teniendo en cuenta la estructura de la cartera crediticia. 


\subsection{Implicaciones del nuevo sistema de cálculo del capital mínimo en el sector PyME en Colombia}

Teniendo en cuenta que el comportamiento de la cartera crediticia otorgada a PyMEs mantiene un comportamiento diferente -en cuanto a niveles de morosidad- comparado con las colocaciones en grandes empresas, en este apartado se pretende evaluar el posible impacto que el nuevo sistema de cálculo de capital mínimo presentado en la sección anterior, puede tener en el acceso al crédito por parte de las pequeñas y medianas empresas en el sistema financiero colombiano.

Para medir este impacto, se divide la sección en dos partes: estimación de la probabilidad de impago de las PyMEs y cálculo de los requerimientos de capital del sistema financiero colombiano de acuerdo con las probabilidades de impago obtenidas, incluidos los niveles de exposición por segmento, MiPyMEs, PyMEs y grandes empresas.

\subsubsection{Aproximación a la probabilidad de impago (PD)}

La segmentación de la cartera por Corporate y Retail Exposures requiere que se analice al sector PyMEs de acuerdo con su tamaño y nivel de financiamiento, con el fin de definir un tope a la hora de clasificar un crédito con alto o bajo grado de exposición. Sin embargo, esta es una de las limitaciones de análisis pues no se dispone de información acerca de la representatividad de operaciones de crédito otorgadas a PyMEs por montos; con lo que se establecen como criterios de clasificación las siguientes hipótesis:

> Retail: se asigna al segmento de microcréditos que corresponden a operaciones cuyo monto no supera los US $\$ 6.500$ dólares (véase Superintendencia Financiera de Colombia, 2008).

DCorporate: se clasifica cartera comercial otorgada a PyMEs.

De igual manera, no se dispone de datos exactos sobre los niveles de morosidad para las PyMEs. A fin de estimar una cifra que se acercara a la realidad colombiana, se parte del indicador de morosidad para PyMEs (3.2\%) estimado por el Banco Mundial para el año 2006 (Banco Mundial, 2008). A partir de esta cifra se extrapola una serie desde 2003 hasta 2007, tomando como evolución del indicador, la tasa de crecimiento de la morosidad del total de la cartera comercial publicada por la Superintendencia Financiera de Colombia.

Para el caso de microempresas y grandes empresas se utilizan los datos de morosidad publicados en el informe de gestión de marzo de 2008 de la Superintendencia Financiera de Colombia. 
Con estos datos se calcula la probabilidad de impago de cada segmento como la media de la serie. Los resultados obtenidos se detallan en la Tabla 2.

Tabla 2. Probabilidad de impago por segmento

\begin{tabular}{|l|c|c|c|}
\hline & Microempresas & PyME & Empresas \\
\hline dic-03 & $5.35 \%$ & $5.72 \%$ & $3.18 \%$ \\
\hline dic-04 & $5.41 \%$ & $4.13 \%$ & $2.29 \%$ \\
\hline dic-05 & $4.56 \%$ & $3.64 \%$ & $2.02 \%$ \\
\hline dic-06 & $5.37 \%$ & $3.20 \%$ & $1.78 \%$ \\
\hline dic-07 & $6.98 \%$ & $4.15 \%$ & $2.31 \%$ \\
\hline $\begin{array}{l}\text { mar- } \\
08\end{array}$ & $8.06 \%$ & $4.90 \%$ & $2.73 \%$ \\
\hline Media & $\mathbf{5 . 5 3 \%}$ & $\mathbf{4 . 1 7 \%}$ & $\mathbf{2 . 3 2} \%$ \\
\hline
\end{tabular}

Fuente.Cálculos propios con datosde la Superintendencia Financiera de Colombia,2008yAnalistas Financieros Intercionales, 2007

Bajo la estimación realizada, se evidencia que la probabilidad de impago es mayor a menor tamaño empresarial del acreditado y que a partir del 2007 aumenta levemente para todas las categorías. Estos resultados son congruentes con análisis de similares características y con el comportamiento del sector bancario colombiano (Saurina y Trucharte, 2002; Banco Mundial, 2008). Esta característica ha sido tenida en cuenta por el sector financiero a la hora de segmentar por perfiles de cliente, por ello se ha incluido en el cálculo del capital mínimo, al plantearse una distinción entre exposiciones Corporate y Retail.

\subsubsection{Cálculo de los requerimientos de capital de acuerdo a la proba- bilidad de impago y grado de exposición por segmento}

Una vez estimada la probabilidad de impago, se estima el capital mínimo requerido por probabilidad de impago y grado de exposición en cada segmento. Sin embargo, no se cuenta con datos históricos de los créditos otorgados al segmento, para lo cual se utilizan las estimaciones realizadas por la firma Analistas Financieros Internacionales (2007), en las cuales se presentan aproximaciones de los volúmenes de crédito por segmento y el nivel de morosidad de las PyMEs y las grandes empresas a cierre de 2006, para los 15 principales bancos del país. Esta información es consistente con los datos sectoriales de las instituciones bancarias que se analizaron en el apartado del sistema bancario. 
Gráfica 2. Porcentaje de créditos otorgados por segmento

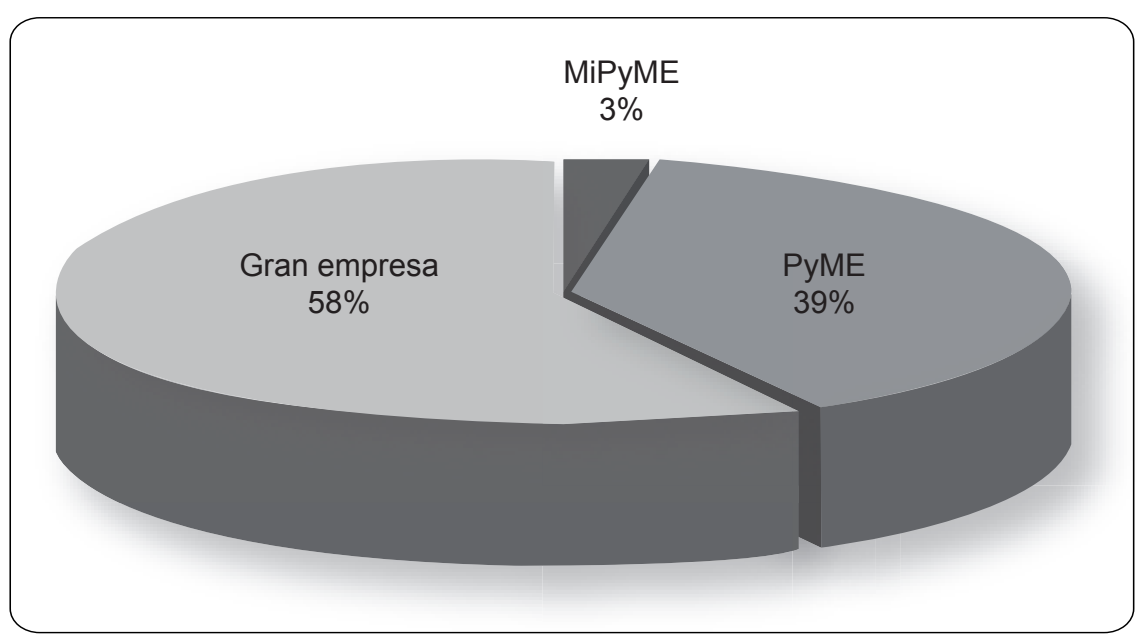

Fuente. Analistas Financieros Internacionales, 2007

A partir de estos datos, se hallan los requerimientos de capital con base en la formulación descrita anteriormente. Los resultados se muestran en la Tabla 3.

Es interesante observar que los nuevos requerimientos de capital bajo Basilea
Il de acuerdo al nivel de morosidad para el segmento PYME y Gran Empresa (definidos dentro de la categoría de Corporate) disminuyen significativamente en comparación con el sistema actual, mientras que los de la categoría Retail aumenta en un $4.3 \%$.

Tabla 3. Requerimientos de capital por segmentos y morosidad

\begin{tabular}{|l|l|l|l|}
\hline & $\begin{array}{l}\text { MiPyME } \\
\text { PD 5.53\% }\end{array}$ & $\begin{array}{l}\text { PyME } \\
\text { PD 4.17\% }\end{array}$ & $\begin{array}{l}\text { Gran empresa } \\
\text { PD 2.32\% }\end{array}$ \\
\hline Enfoque IRB Corporate (01/2002) & NA & $4.5 \%$ & $1.8 \%$ \\
\hline Enfoque IRB Retail $(01 / 2002)$ & $12.3 \%$ & NA & NA \\
\hline Enfoque estándar & $8 \%$ & $8 \%$ & $8 \%$ \\
\hline Sistema actual & $9 \%$ & $9 \%$ & $9 \%$ \\
\hline
\end{tabular}

PD : Probabilidad de Default

Fuente. Cálculos propios con datos de la Superintendencia Financiera de Colombia

La información obtenida permite calcular los requisitos de capital a partir del grado de exposición por sector. La suma de los requerimientos individuales se utilizará para estimar los requerimientos de capital total del sector. 
Para su cálculo, se multiplica el porcentaje de exposición de cada segmento por la probabilidad de impago. El resultado obtenido se compara con el enfoque estándar y enfoque actual. Finalmente, los cálculos realizados arrojan las siguientes cifras:

Tabla 4. Requerimientos de capital de acuerdo con el nivel de morosidad y grado de exposición por segmento

\begin{tabular}{|l|l|l|l|l|}
\hline & Micro & PyMEs & Empresas & Total \\
\hline $\begin{array}{l}\text { Morosidad } \\
\text { promedio }\end{array}$ & $5.53 \%$ & $4.17 \%$ & $2.32 \%$ & \\
\hline $\begin{array}{l}\text { Porcentaje de } \\
\text { Exposición }\end{array}$ & $2.9 \%$ & $38.9 \%$ & $58.3 \%$ & $100.0 \%$ \\
\hline $\begin{array}{l}\text { Req Cap (Enf } \\
\text { IRB) }\end{array}$ & $0.35 \%$ & $1.77 \%$ & $1.02 \%$ & $3.1 \%$ \\
\hline $\begin{array}{l}\text { Req Cap (Enf } \\
\text { Estándar) }\end{array}$ & $0.23 \%$ & $3.1 \%$ & $4.7 \%$ & $8.0 \%$ \\
\hline $\begin{array}{l}\text { Req Cap (Sist } \\
\text { Actual) }\end{array}$ & $0.26 \%$ & $3.50 \%$ & $5.24 \%$ & $9.0 \%$ \\
\hline
\end{tabular}

Fuente: Cálculos propios

Se encuentra entonces que los requerimientos de capital aumentan bajo el nuevo enfoque para el crédito MiPyME, mientras que son las PyMEs y las grandes empresas las que se ven favorecidas bajo el nuevo método, en razón a que se liberan flujos retenidos al disminuir el capital requerido por morosidad y grado de exposición.

\subsubsection{Basilea II y requerimientos de capital en crédito PyME: resultados}

Ahora bien, si por una parte aumentan las necesidades de capital (MiPyME) y por otra disminuyen (PyME y gran empresa) ¿Cuál es el saldo en términos de flujo de capital requerido para el sector bancario?.

Para estimar este resultado neto, bajo el enfoque actual (establecido por la Superintendencia Financiera) y el nuevo, se calcula la diferencia entre los requerimientos y se multiplica por el total de la cartera en cada segmento. Los resultados obtenidos son los que se muestran en la siguiente tabla. 
Tabla 5. Liberalización de capital bajo Basilea II

\begin{tabular}{|c|c|c|c|c|}
\hline \multicolumn{5}{|c|}{ Saldo en requerimientos de capital } \\
\hline & Mypimes & PyMEs & Gran empresa & Total \\
\hline Req Cap (Enf IRB) & $0,35 \%$ & $1,77 \%$ & $1,02 \%$ & $3,1 \%$ \\
\hline Req Cap (Sist Actual) & $0,26 \%$ & $3,50 \%$ & $5,24 \%$ & $9,0 \%$ \\
\hline Diferencial requerimientos & $-0,09 \%$ & $1,73 \%$ & $4,22 \%$ & $5,86 \%$ \\
\hline Cartera total (MM U\$) & $\$ 1.020$ & 9.215 & 25.689 & \\
\hline Saldo (MM U\$) & $-\$$ & 160 & $\$ \quad 1.084$ & 1.243 \\
\hline
\end{tabular}

Fuente. Cálculos propios con datos de la Superintendencia Financiera de Colombia

Bajo las estimaciones realizadas, el nuevo enfoque favorece de manera sustancial a las entidades financieras y en particular al segmento PyME y gran empresa, porque se liberan recursos que pueden destinarse a aumentar la cartera de estos segmentos. Bajo estos términos los resultados son una noticia alentadora para las PyMEs. El hecho de que se reduzcan los niveles de cubrimiento podría ser un incentivo para que las entidades de crédito incrementen los niveles de colocación en este sector.

A la par, es evidente el hecho de que el sistema actual castiga fuertemente a la cartera de grandes empresas, al obligar a cubrir en un $9 \%$ su nivel de exposición, tasa que al compararse con el nuevo esquema, resulta muy superior, sobre todo, si se tiene en cuenta que su morosidad es baja.

Sin embargo, el segmento MiPyMEs presenta un aumento en los requerimientos de capital bajo el nuevo esquema, que es importante considerar al evaluar el impacto en la oferta crediticia a este segmento.

Por otra parte, es importante aclarar que la clasificación supuesta en términos de grado de exposición establece un escenario optimista para el cálculo de los requerimientos de capital, debido a que el segmento de gran exposición (Retail) está subestimado al establecer en esta categoría solamente los microcréditos, que si bien tienen los porcentajes de impago más altos por segmento, poseen la participación más baja en el total de cartera comercial. Así, en la medida en que se aumente el monto de clasificación por exposición, reduciendo la cantidad de créditos tipo Corporate, aumentarán (ceteris paribus) los requerimientos de capital.

En conclusión, el hecho de que Colombia maneje niveles bajos de morosidad y muestre un comportamiento positivo en 
el control del impago, plantea la favorabilidad de acoger las disposiciones del nuevo acuerdo frente al esquema estándar. El buen comportamiento de las PyMEs en cuanto al cumplimiento de sus obligaciones permitirá mantener bajo el capital requerido, beneficiando los requerimientos de capital de las instituciones financieras y posibilitando el camino para evaluar nuevas alternativas en la profundización del mercado de crédito PyME en Colombia.

\section{Conclusiones}

B instituciones financieras colombianas y hace necesario estructurar y diseñar una gestión de riesgos integral, que permita controlar y establecer límites en aras de generar una estabilidad financiera en el largo plazo. Sin embargo su aporte más destacable, es que bajo la favorable situación por la que atraviesa Colombia y los buenos resultados en términos de morosidad, el Nuevo Acuerdo de Capitales plantea oportunidades al crédito PyME, pues libera flujos de capital al recalcular el capital mínimo requerido por segmento.

Esto, por lo tanto, permitirá a los bancos profundizar en este segmento, con el fin de dar apoyoal crecimiento de las pequeñas y medianas empresas. 


\section{Bibliografía}

Analistas Financieros Internacionales (2007). Desarrollo del mercado de Titularización de Cartera de PYMES en Colombia.

Asociación Nacional de Instituciones Financieras - ANIF - (2007). Sistema Financiero Guía Empresarial, pgs 32-34.

Asociación Nacional de Instituciones Financieras - ANIF - (2007). La gran encuesta PyME. Informe de resultados primer semestre de 2007. ANIF Centro de Estudios Económicos.

Banco Mundial (2008). Colombia, Financiamiento bancario para las pequeñas y medianas empresas (PyME), Oficina Regional para Latinoamérica y el Caribe, Unidad de Gestión para México y Colombia.

Cárdenas, M. y Mejía, C. (2007). Informalidad en Colombia: Nueva Evidencia, Documento de trabajo No. 35, Fedesarrollo.

Clavijo, S. (2006). Evaluación, retos y perspectivas de las PYMEs en Colombia", Asociación Nacional de Instituciones Financieras - ANIF -

Clavijo, S. (2008). Coyuntura PyME $\| \cdot 2008$. Asociación Nacional de Instituciones Financieras -Anif-.

Comisión Económica para América Latina -CEPAL- (2006). Crecimiento Económico de América Latina y el Caribe 2006. 
Conpes (2007). Documento 3484: Política Nacional para la transformación productiva y la promoción de las Micro, Pequeñas y Medianas Empresas: un esfuerzo públicoprivado.

Departamento Administrativo Nacional de Estadística (2005). Censo 2005.

Duff \& Phelps de Colombia (2008). Establecimientos de crédito en Colombia.

Sandoval, C. (2007). Alternativas de financiamiento para el desarrollo de las PyMEs: el caso del factoring". Asobancaria.

Saurina, J y Trucharte, C. (2008). Las pequeñas y medianas empresas en el sistema crediticio español y su tratamiento según Basilea II. Banco de España. 